\title{
A Systematic Literature Review of Reviews on the Effectiveness of Chlamydia Screening
}

William Chi Wai Wong, Stephanie Tsz Hei Lau, Edmond Pui Hang Choi, Joseph D Tucker, Christopher K Fairley, and John M Saunders

Correspondence to Dr. Edmond Pui Hang Choi, School of Nursing, LKS Faculty of Medicine, the University of Hong Kong, 21 Sassoon Road, Pokfulam, Hong Kong (e-mail: h0714919@connect.hku.hk ) (phone: +852 3917 6972) (fax: +852 28726079)

Author affiliations: Department of Family Medicine and Primary Care, LKS Faculty of Medicine, The University of Hong Kong, Hong Kong (William Chi Wai Wong, and Stephanie Tsz Hei Lau); Department of General Practice, The University of Hong Kong-Shenzhen Hospital, Shenzhen, China (William Chi Wai Wơng); School of Nursing, LKS Faculty of Medicine, The University of Hong Kong, Hong Kong (Edmond Pui Hang Choi); Division of Infectious Diseases, Department of Medicine, UNC School of Medicine, University of North Carolina at Chapel Hill, ChapelHill, United States (Joseph D Tucker); Faculty of Infectious and Tropical Diseases, The London School of Hygiene \& Tropical Medicine, London, United Kingdom (JosephD Tucker); Melbourne Sexual Health Centre, Alfred Health, Melbourne, Victoria, Australia (Christopher K Fairley); Central Clinical School, Monash University,

(C) The Author(s) 2019. Published by Oxford University Press on behalf of the Johns Hopkins Bloomberg School of Public Health. All rights reserved. For permissions, please e-mail: journals.permissions@oup.com. 
Melbourne, Victoria, Australia (Christopher K Fairley); and UCL Centre for Clinical Research in Infection and Sexual Health, Institute for Global Health, London, United Kingdom (John M Saunders)

This work was funded by Seed Fund for Basic Research (Project number: 104004734), The

Conflict of interest: none declared

Running head: Review on the Effectiveness of Chlamydia Screening

University of Hong Kong, Hong Kong

Renting Review 


\begin{abstract}
Chlamydia trachomatis is the most common bacterial sexually transmitted infection, causing significant morbidity and economic burden. Strategies like national screening programs or hometesting kits were introduced in some developed countries, yet their effectiveness remains controversial. This systematic review examined reviews of chlamydia screening interventions to assess their effectiveness and the elements that contribute to their success to guide public policy and future research. The review assessed English material published after year 2000 in PubMed, Cochrane Library, the British Nursing Index, Medical Database, and Sociological Abstract, in addition to World Health Organization Global Health Sector Strategies, the European Center for Disease Prevention and Control guidelines, and PROSPERO. Systematic reviews that focused on chlamydia screening interventions were included. Using the socio-ecological model, we examined the levels of interventions that may affect the uptake of chlamydia screening. 19 systematic reviews were included. Self-collection in home-testing kits significantly increased screening among females 14-50years of age. At the organizational level, using electronic health records and not creating additional costs facilitated testing. At the community level, outreach interventions in community/parent centers and homeless shelters reached high screening rates. At the policy level, interventions with educational and advisory elements could result in significant improyements in screening rates.
\end{abstract}

Key words: Sexually transmitted infections; Chlamydia; Screening; Effectiveness; Review Abbreviation:

PID: pelvic inflammatory disease 


\section{Introduction}

Chlamydia trachomatis is the most common bacterial sexually transmitted infection and causes significant morbidity and economic burden globally ${ }^{1}$. There are approximately 131 million new cases of chlamydial infection occurring in individuals aged 15-49 years annually, with an incidence rate of 38 per 1000 females and 33 per 1000 males. Among the non-viral sexually transmitted infections, chlamydia is the most costly infection, which costs the US healtheare system approximately US\$516 million annually ${ }^{2}$

The majority of chlamydia infections are asymptomatic with only $30 \%$ of women and $10 \%$ of men develop symptoms ${ }^{3}$. Therefore, infected people often do not seek testing and are unaware of their infection ${ }^{4}$. The symptoms of uncomplicated chlamydia infection in women include abnormal vaginal discharge and post-intercourse bleeding ${ }^{1}$. Chlamydia trachomatis is also an important cause of pelvic inflammatory disease (PID) ${ }^{5}$. A community-based study found that the annual incidence of PID among women with untreated chlamydia was about $10 \%{ }^{6}$. Other reproductive sequelae of chlamydia include infertility, ectopic pregnancy and chronic pelvic pain 5 . The reported infertility rate after one episode of PID was $8 \%$ and it increases to $38 \%$ after three PID episodes ${ }^{7}$. Chlamydia infection is also associated with negative psychosocial impact and poor sexual quality of life in young women ${ }^{8,9}$.

To reduce the burden of chlamydia control strategies a number of developed countries have implemented national screening programs ${ }^{10}$, opportunistic screening for women $<25$ years ${ }^{11,12}$, and targeted screening ${ }^{13}$. England initiated their National Chlamydia screening program where free screening is provided in settings such as genitourinary medicine clinics, general practices, 
and community pharmacies. For sustainable benefits to occur, screening and follow-ups should be regular ${ }^{14}$. Such requirements may be difficult as the target population must utilize health service regularly and health providers must offer the tests at appropriate intervals. Administrative systems also need to track individuals who attend various health venues ${ }^{15}$. The introduction of quick and non-invasive testing methods such as nucleic acid amplification tests has allowed testing to be done in more convenient and out-of-clinic settings however chlamydia testing rates remain sub-optimal ${ }^{16}$. Barriers that lead to continued low screening rates include hesitation and embarrassment from patients and limited understanding and training amongst healthcare providers $^{17,18}$.

There is an abundance of literature relating to improying the rates of chlamydia screening. Certain interventions target patients while others target the healthcare providers. The heterogeneity of chlamydia screening interventions make clinicians and researchers difficult to determine which are effective. Hence there is a lack of consensus on the most effective way to increase chlamydia screening. To address this issue we carried out a systematic review of systematic review articles, of chlamydia screening, in order to guide our public health policy and research in the area.

\section{Methods}

We conducted this systematic review of systematic reviews in accordance with the guideline of the Preferred Reporting Items for Systematic Reviews and Meta-Analysis (PRISMA). The systematic review protocol was registered on the PROSPERO database (registration number: CRD42018085514) 


\section{Information Sources and Search strategy}

Five databases were used to identify review articles that have evaluate chlamydia screening intervention. They are PubMed, Cochrane Library, the British Nursing Index, Medical Database, and Sociological Abstract via ProQuest. Searches in PubMed were conducted with the Medical Subject Headings (MeSH) terms of "Chlamydia" and "mass screening" then filtered for systematic reviews and meta-analysis. In the Cochrane library, the advanced seareh function was used for the key words "chlamydia" and "screening". In ProQuest, advanced search was carried out for the key words "chlamydia", "mass screening", and "systematic review".

Besides, the reference lists of the World Health Organization Global Health Sector Strategies and the European Center for Disease Prevention and Control guidelines were screened. PROSPERO database was also searched. Experts in our network were approached for suggestions of relevant papers.

\section{Inclusion Criteria}

We only included systematic reviews and meta-analysis published in English after 2000 because nucleic acid amplification test diagnostic technology was not introduced until then. Interventions must focus on Chlamydia screening (studies regarding sexually transmitted infection screening were accepted as long as chlamydia is included). Interventions must have one or more of these outcome measures: number of chlamydia tests, testing rate, retesting rate, and/or treatment rate of chlamydia. 


\section{Screening}

One author (Stephanie Lau) screened the titles and abstracts and two authors (Stephanie Lau and Edmond Choi) independently conducted full text screening and data extraction. Discrepancies were resolved through discussion and input from another author (William Wong).

\section{Data Extraction}

A raw data extraction sheet was created and included information such as target populations, settings, type of testing, number of people tested, prevalence, number of people treated, effectiveness, and barriers. The data extraction summary sheet is available in the appendix.

\section{Quality Assessment}

The quality of the included papers was assessed using the validated scale Assessment of Multiple Systematic Reviews (AMSTAR). It provides a 16-item checklist on the quality of systematic review methodology.

\section{Analysis}

The socio-ecological model was used to organize the interventions (Figure 1). The socioecological model is a systems model with multiple levels of influence including interpersonal, organizational, community, and policy levels. Interventions based on the individuals' characteristics aim to influence their knowledge, attitude, and beliefs towards a health behavior. Interventions at the interpersonal level influence social norms to overcome individual barriers. Involved parties include friends, family, community health workers, and health service providers. Interventions in the organizational level involve healthcare systems, health 
departments, and health clinics. Interventions in the community level try to leverage resources and encourage the participation of community-level institutions. Interventions at the policy level aim to create systemic changes. Health intervention developers often utilize this model to ensure that the intervention accounts for these interwoven bands of influence to result in the greatest impact ${ }^{19}$.

\section{Results}

200 studies were identified in the initial search of the databases and an additional 5 studies were identified through other sources. During the title screen, 148 studies were excluded because their titles did not mention that it was a systematic review, was not about sexually transmitted infections, or was not in English. During the abstract screen, 33 further studies were excluded because the abstract did not mention anything related to chlamydia screening and testing. 24 reviews were assessed by full text screen in which five was excluded to result in the final inclusion of 19 reviews. The study detaits are shown in Web Table 1 One study was excluded because it described itself as a review but was ultimately not a systematic review. Two studies were excluded because it was regarding diagnostic tools for detecting chlamydia. Two studies were excluded because it was about rescreening (Figure 2).

\section{Quality Assessment}

Papers were marked out of a score of 16 and categorized into groups of low quality (0-5.5 score), medium quality (6-11.5 score), and high quality ( $\geq 12$ score). Eleven systematic reviews were of low quality, nine of medium quality, and one of high quality. Twelve papers did not define selection criteria of studies. Two papers provided a list of excluded studies with justifications 
and one provided the list without justifications. One review reported funding information. Four utilized a satisfactory risk of bias technique for included studies while one obtained a "partial yes" in this criterion.

\section{Individual and Interpersonal Levels}

The systematic review by Chacko et al. focused on both health provider-based and client initiated chlamydia screening practices in asymptomatic young women in the United States ${ }^{20}$.

The review revealed that the screening of sexually active women under the age of 20 was the top priority. However, the authors of the review pointed out that it wás challenging to evaluate the effectiveness of health provider-based screening programs because of the large size of program, variations in prevalence rates in populations targeted and the criteria used to determine effectiveness ${ }^{20}$. Besides, the review found that there was a marked absence of programs and no publication describing intervention to foster client-initiated screening behaviors among asymptomatic young women in the United States, despite of the Centers for Disease Control and Prevention's recommendations ${ }^{20}$.

A systematic review by Odesanmi et al. compared the screening uptake levels of home-based self-sampling and clinic-based specimen collection for Chlamydia trachomatis in females aged $14-50$ years old ${ }^{21}$. The review only included randomized control trials. Self-collection is where people bring a kit home and send the sample to a testing facility without needing to attend a health facility ${ }^{21}$. The review supported the use of home sampling to increase screening uptake but cost-effectiveness should be further investigated ${ }^{21}$. Furthermore, the review reported a significant preference for self-collection compared to clinic based testing. Another systematic 
review only focused on the home-based screening strategy and divided home-based screening into seven groups according to test kit and specimen delivery methods. The highest median specimen return rate was in outreach programs in which participants were approached at home with immediate collection of specimens (96.5\%), programs providing home testing kits only on invitation acceptance (78.9\%), home testing kits sent along with an invitation (32.9\%), home testing kits requested without an invitation (31.8\%), home testing kit offered in person (21.4\%), and home testing kits picked up at specific locations $(18.6 \%)^{22}$. However, the interventions targeted a wide range of target populations hence comparison between each program may not be valid. Nonetheless, this review shows that self-collection programs have been conducted in a variety of countries and with different delivery methods, suggesting that home-based testing is a feasible and acceptable chlamydia screening method. However, further studies regarding its costeffectiveness are needed $^{22}$.

\section{Organizational Level}

A systematic review by Taylor et al. categorized screening interventions into levels of effectiveness as measured by the absolute difference in percent of target population screened. Effective and low-cost interventions (<USD\$1,000) included strategic placement of specimen collection materials, routine consultation collection, and electronic health records use. Effectives and low-moderate costs interventions ( $\$$ USD $<1,001-10,000)$ utilized patient reminder strategies such as postcards and calls. Effective and high costs (\$USD\$10,001-100,000) interventions inyolved dedicated screening staff. The authors concluded there is a variety of effective and costeffective screening interventions ${ }^{23}$. 
Interventions targeting females resulting in significant increases in screening rates include linking screening to pap smears (6.9\% vs. $4.5 \%)$, computer alerts for doctors (15.5\% vs. $12.4 \%)$, and free sexual health consultation $(16.8 \% \text { vs. } 13.2 \%)^{24-26}$. Screening program initiation is associated with chlamydia infection reduction and screening women with a certain range of risk factors reduces PID incidence for 1-year ${ }^{27,28}$. Challenges in implementing healthcare provider screening interventions include lack of protocols of urine sample obtainment, insufficient knowledge about chlamydia and urine-based tests, and reluctance of staff to be engaged in adolescent sexually transmitted infection screening ${ }^{29}$. The effectiveness and feasibility of screening for chlamydia in emergency departments has also been examined ${ }^{30}$. Prevalence of emergency department patients was high $(9.1-9.5 \%)^{28,31}$. Barriers include finding a location for screening in the emergency department, clinician's witlingness and time constraints, and availability of other staff ${ }^{23,32-36}$.

The systematic review by Gudka etal. examined pharmacy-based screening interventions ${ }^{37}$. Return rates were high in studies with $47 \%$ in England, $63.9 \%$ in Scotland, $28 \%$ in Australia, and $38 \%$ in the United States ${ }^{38-41}$. Major barriers of pharmacy-based screening included having to return the specimen to designated sites and lack of privacy ${ }^{37,41}$ for patients and increased workload or feeling uncomfortable when offering the test, and lack of in store advertising for pharmacists 42 . The major benefits for patients included convenience, anonymity and no need for appointment, affordability ${ }^{36}$, shorter waiting times, and friendly non-judgmental attitude of pharmacists ${ }^{43}$. It was concluded that chlamydia screening in community pharmacies were feasible and could be a convenient option ${ }^{43}$. 


\section{Community Level}

In a review by Bernstein et al., screening interventions in non-clinic settings were examined ${ }^{44}$. The review revealed that high number of tests could be carried out in correctional settings and identified a large amount of asymptomatic infections. The total number of people approached was unavailable but high prevalence was found amongst adolescent girls in juvenile detention centers in Southern City (24.7\%), Georgia (16.8\%), and California $(13.0 \%){ }^{45}$. Other communitybased screening included homeless shelters, family court, and mobile van but they identified few new infections ${ }^{46-48}$. High chlamydia prevalence was also found when screening in educational settings ${ }^{49}$. The prevalence was $12.4 \%$ for males and females in a classroom-based strategy, $18.8 \%$ for females in school-based health clinic, $15.6 \%$ for male and females in other school locations, and $15.0 \%$ in clinical session screening ${ }^{47,50-52}$. Chlamydial screening in educational settings is a feasible approach with a range of deliverymethods identifying large numbers of infected adolescents ${ }^{53}$.

In the systematic review by Hengel et al., outreach interventions targeting young people aged 1529 years, men who have sex with men, and sex workers were identified ${ }^{53}$. Amongst the outreach settings, the highest participation rate was in community venues such as community centers, parenting centers, and homeless shelters (81.4\%) and social venues such as sport venues or bars $(80.4 \%)$. In interventions targeting adolescents and young adults, Gold et al. found a high testing rate $(75.2 \%$, tests $=92)$ in a screening program in a football club changing room in Australia ${ }^{54}$. High testing rates were also found in community venues targeting dropouts, new immigrants, and vocational school students in the Netherlands $(79.6 \% \text {, tests }=74)^{52}$. Low participation rates were identified in street and public community areas (median $=23.9 \%, \mathrm{n}=3$ ) and sex venues 
$(10.4 \% \text { and } 24.3 \%, \mathrm{n}=2)^{55}$. The authors concluded that chlamydial outreach programs are able to result in high participation rates but with limited reach. Settings that resulted in higher participation rates seem to be less public ${ }^{55}$.

\section{Policy Level}

\section{Education and advisory}

In the systematic review by Ginige et. al., a cluster randomized control trialassessing the effectiveness of an educational package in general practitioners in Belgium was analyzed ${ }^{18}$. The target population was females aged below 35 years old ${ }^{56}$. The intervention included a stimulated consultation and text messages regarding communication skills. The intervention group performed significantly better by carrying out more screening (median 6 patients per general practitioner vs. 3 patients per general practitioner, $p=0.035)^{57}$. Another study investigated the effectiveness of having a health advisor in primary healthcare centers to increase awareness of chlamydia and to train the staff in Scotland. Testing rates in the intervention centers was significantly higher than those in the control $(120 \%$, vs $11 \%, \mathrm{p}=<0.001)$. However, the denominator value indicating the number of patients seen was missing in the primary paper ${ }^{55}$.

Allison et al. carried out a study regarding the effectiveness of an internet-based continuing medical education to increase chlamydia screening by primary care physicians in the US. The continuing medical education course consisted of 4 modules over 3 months. The chlamydia screening rates for before, during and after the intervention were $16.2 \%, 13.3 \%$, and $15.5 \%$ for the intervention group respectively vs. $18.9 \%, 13.0 \%, 12.4 \%$ for the control $(\mathrm{p}=0.044$ for postintervention differences) ${ }^{27}$. 


\section{System-level changes in clinical practice}

In the same systematic review by Ginige et al. a randomized control trial assessing the effectiveness of a multi-phased intervention aimed to result in system-level changes in clinical practice regarding chlamydia screening was analyzed ${ }^{43}$. The intervention consisted of four stages where the first stage was engagement with the organization's leaders to investigate the discrepancy between current and best practice. The second stage involved the formation of adolescent care teams, a model for practice change, and a staff toolkit. The third stage involved monthly meetings between clinic team members. The final stage developed performance indicators. The intervention clinics saw a significant number of fémales ages 14-18 screened as compared to the control clinics (478 of 1017 vs 203 of 1194, p<0.001) ${ }^{47}$. The authors concluded that there is a variety of ways to increase chlamydia sereening but more randomized control trials are needed.

\section{Discussion}

Adolescent girls have always been the targets of screening programs but high chlamydial prevalence was also found in adolescent boys ${ }^{58,59}$. Screening all or only high-risk adolescents have strong implications on monetary and human resources. Even if mass screening for adolescents is implemented, it is difficult to ensure the acceptance of the target population. The Centers for Disease Control and Prevention in the United States has recommended annual screening of women under age 26 since 1993 but less than half was screened in $2012^{44,60}$. Atrisk individuals such as women, adolescents, and those entering juvenile detention centers are often missed due to the lack of awareness in healthcare providers and limited resources ${ }^{45}$. Young women entering correctional services are at higher risk of chlamydia and high prevalence of 
chlamydia has been found ${ }^{20,46,59}$. The Centers for Disease Control and Prevention has recommended for the universal screening of females during intake in juvenile correctional services and screening rates increased from $55 \%-58 \%$ from $2005-2008{ }^{21}$. Therefore, the questions are how to promote chlamydia infection awareness; how to reach the target population; and what environment changes encourage screening.

While asymptomatic individuals are unlikely to obtain a routine check-up, clinicians also admitted to being hesitant in screening sexually active asymptomatic females ${ }^{61}$. High-risk population should be linked to different health contacts such as páp smears, sexual health consultations or in pharmacy. Self-collection was found to significantly increase uptake of screening and was preferred over clinic-based testing in women aged $14-50$ years ${ }^{62}$. Selfcollection strategies should be promoted to adolescents via social media because social media is a popular portal for teenagers to exchange and share information ${ }^{63}$. Social media interventions have been shown to be effective in significantly increasing syphilis and human immunodeficiency viruses testing in youth and in promoting human immunodeficiency viruses testing in men who have sex with men ${ }^{15,55}$. However, the effectiveness of social media interventions has not been demonstrated for chlamydia testing. This calls for further research in social media campaigns to increase chlamydia testing amongst adolescents. Another approach is to implement structural changes to alter the attitudes of healthcare providers in that they make testing adolescents a priority. Healthcare providers should also receive proper training so that they are able to approach adolescent patients confidently for chlamydia screening. Structural interventions mentioned previously did not focus on adolescents hence development of adolescent focused programs is needed ${ }^{64}$. 
In essence, we want to know whether screening interventions are able to reduce chlamydia's disease burden. Some studies revealed that chlamydia screening reduced chlamydia infections and that screening women with a certain range of risk factors reduced PID incidence, other studies claim a lack of evidence in supporting opportunistic screening in the general population below 25 years of age ${ }^{65,66}$. While our review identified a large number of studies of chlamydia screening programs, most studies did not report data on chlamydia associated morbidity. We are not able to determine the impact of chlamydia screening on chlamydia assocíated morbidity. As we relied on the analysis and data presented by primary authors, this exclusion is sub-optimal. The lack of evidence on the effectiveness of chlamydia screening programs in reducing disease burden has caused several countries to focus on case identification and management ${ }^{67}$. A recent review has suggested a shift from an infection-based focus to increase screening uptake in asymptomatic populations, to a health outcomes-based focus to improve case detection among high-risk populations and case management ${ }^{66}$. Greater emphases need to be on strategies for infected individuals to minimize re-infection such as improving partner notification, treatment of sexual partners or re-testing $\stackrel{67}{2}$

Our methodology has a few limitations. Firstly, it depended on the inclusion and analysis of primary studies by the review's authors. Secondly, included reviews differed significantly in terms of interyention design, target populations, settings and study outcomes. Therefore, the heterogeneity of the included systematic reviews has made comparison of effectiveness difficult.

Apart from screening coverage and prevalence, other data regarding treatment, partner management, and retesting which is vital in determining its success, was often unavailable. Lastly, the results are presented without focus on the contextual nature of the studies. Factors 
such as cultural attitudes, government policies, and differences in healthcare system could have effects on the outcomes.

\section{Conclusion}

There is evidence that shows the most important factor that determines the level of chlamydia screening was age. Therefore, national programs should focus on young males and females in the general population rather than individuals with high-risk characteristics. Reninders and outreach in certain community settings increased screening, but further information regarding costeffectiveness is needed. Four interventions at the policy level were found to be effective; while the interventions differ, they share similar educational, advisory, and supervisory elements. Structural interventions are vital in changing the attitudes and awareness of chlamydia screening to result in systemic change. Therefore, initiatives to train healthcare providers in carrying out appropriate testing for adolescents is needed.

\section{Acknowledgements:}

This work was funded by SeedFund for Basic Research (Project number : 104004734), The University of Hong Kong. Hong Kong

Conflict of interest: none declared 


\section{References:}

1. World Health Organization. WHO Guidelines for the Treatment of Chlamydia trachomatis. Geneva, Switzerland: World Health Organization; 2016. https://www.who.int/reproductivehealth/publications/rtis/chlamydia-treatmentguidelines/en/. Accessed February 12, 2019.

2. Owusu-Edusei Jr K, Chesson HW, Gift TL, et al. The estimated direct medical cost of selected sexually transmitted infections in the United States, 2008. Sexually Transmitted Diseases. 2013;40(3):197-201.

3. Jackson LJ, Auguste P, Low N, Roberts TE. Valuing the health states associated with Chlamydia trachomatis infections and their sequelae: a systematic review of economic evaluations and primary studies. Kalue in Health. 2014;17(1):116-130.

4. Manavi K. A review on infection with Chlamydia trachomatis. Best Practice \& Research Clinical Obstetrics \& Gynaecology. 2006;20(6):941-951.

5. Wiesenfeld HC. Screening for Chlamydia trachomatis infections in women. New England Journal of Medicine. 2017;376(8):765-773.

6. Oakeshott P, Kerry S, Aghaizu A, et al. Randomised controlled trial of screening for Chlamydia trachomatis to prevent pelvic inflammatory disease: the POPI (prevention of pelvic infection) trial. BMJ. 2010;340:c1642.

7. Weström L, Joesoef R, Reynolds G, Hagdu A, Thompson SE. Pelvic inflammatory disease and fertility. A cohort study of 1,844 women with laparoscopically verified disease and 657 control women with normal laparoscopic results. Sexually Transmitted Diseases. 1992;19(4):185-192. 
8. Cai T, Mondaini N, Migno S, et al. Genital Chlamydia trachomatis infection is related to poor sexual quality of life in young sexually active women. The Journal of Sexual Medicine. 2011;8(4):1131-1137.

9. Kangas I, Andersen B, Olesen F, Møller JK, Østergaard L. Psychosocial impact of Chlamydia trachomatis testing in general practice. BrJ Gen Pract. 2006;56(529):587-593.

10. LaMontagne D, Fenton K, Randall S, Anderson S, Carter P. Establishing the National Chlamydia Screening Programme in England: results from the first full year of screening. Sexually Transmitted Infections. 2004;80(5):335-341.

11. Department of Health. Chlamydia trachomatis: summary and conclusions of CMO's expert advisory group. London, the United Kingdom: Department of Health; 1998.

12. Royal Australian College of General Practitioners. Guidelines for preventive activities in general practice. 6th ed. Melbourne, Australia: Royal Australian College of General Practitioners; 2005.

13. Australian Government Department of Health and Ageing. Clinical Practice Guidelines: Antenatal Care - Module 1. Canberra, Australia: Australian Government Department of Health and Ageing; 2012. https://consultations.health.gov.au/phdtobacco/chinical-practice-guidelines-antenatal-caremódule/supporting_documents/ANC_Guidelines_Mod1FINAL\%20D13871243.PDF Accessed February 12, 2019.

14. Peto J, Gilham C, Fletcher O, Matthews FE. The cervical cancer epidemic that screening has prevented in the UK. The Lancet. 2004;364(9430):249-256. 
15. Low N, Bender N, Nartey L, Shang A, Stephenson JM. Effectiveness of chlamydia screening: systematic review. International Journal of Epidemiology. 2008;38(2):435-448.

16. Fairley CK, Hocking J, Gunn J, Chen MY. No barriers to chlamydia testing in sexually active young women. Medical Journal Of Australia. 2005;183(10):548-549.

17. Family Planning Victoria. Review of sexual health clinical services in Victoria. Melbourne, Australia: Family Planning Victoria ;2004.

18. Verhoeven V, Avonts D, Vermeire E, Debaene L, Van Royen P. A short educational intervention on communication skills improves the quality of screening for Chlamydia in GPs in Belgium: a cluster randomised controlled trial. Patient Education and Counseling. 2005;57(1):101-105.

19. Centers for Disease Control and Prevention. Social Ecological Model. https://www.cdc.gov/cancer/nbccedp/sem.htm. Updated January 28, 2013. Accessed February 12, 2019.

20. Chacko MR, Wiemann CM, Smith PB. Chlamydia and gonorrhea screening in asymptomatic young women. Journal of Pediatric and Adolescent Gynecology. 2004;17(3);169-178.

21. Odesanmi TY, Wasti SP, Odesanmi OS, Adegbola O, Oguntuase OO, Mahmood S. Comparative effectiveness and acceptability of home-based and clinic-based sampling methods for sexually transmissible infections screening in females aged 14-50 years: a systematic review and meta-analysis. Sexual Health. 2013;10(6):559569. 
22. Jamil MS, Hocking JS, Bauer HM, et al. Home-based chlamydia and gonorrhoea screening: a systematic review of strategies and outcomes [electronic article]. $B M C$ Public Health. 2013;13(1):189.

23. Taylor MM, Frasure-Williams J, Burnett P, Park IU. Interventions to improve sexually transmitted disease screening in clinic-based settings. Sexually Transmitted Diseases. 2016;43(2S):S28-S41.

24. Bowden FJ, Currie MJ, Toyne H, et al. Screening for Chlamydia trachomatis at the time of routine Pap smear in general practice: a cluster randomised controlled trial. Medical Journal of Australia. 2008;188(2):76-80.

25. Walker J, Fairley CK, Walker SM, et al. Computer reminders for Chlamydia screening in general practice: a randomized controlled trial. Sexually Transmitted Diseases. 2010;37(7):445-450.

26. Scholes D, Grothaus L, McClure et al. A randomized trial of strategies to increase chlamydia screening in young women. Preventive Medicine. 2006;43(4):343-350.

27. Shafer M-AB, Tebb KR, Pantell RH, et al. Effect of a clinical practice improvement intervention on Chlamydial screening among adolescent girls. JAMA. 2002;288(22):2846-2852.

28. Jenkins WD, Zahnd W, Kovach R, Kissinger P. Chlamydia and gonorrhea screening in United States emergency departments. Journal of Emergency Medicine. 2013;44(2):558-567.

29. Stefanski P, Hafner JW, Riley SL, Sunga KL, Schaefer TJ. Diagnostic utility of the genital Gram stain in ED patients. The American Journal of Emergency Medicine. 2010;28(1):13-18. 
30. Kelly JJ, Dalsey WC, McComb J, Njuki F. Follow-up Program for Emergency Department Patients with Gonorrhea or Chlamydia. Academic Emergency Medicine. 2000;7(12):1437-1439.

31. Irvin CB, Nowak B, Moore M, Flynn K, Vretta C. Emergency department Chlamydia screening through partnership with the public health department. Academic Emergency Medicine. 2009;16(11):1217-1220.

32. Gift TL, Irwin KL. Factors that influence the cost effectiveness of gonorrhea screening in emergency departments. Sexually Transmitted Diseases. 2005;32(7):437-438.

33. Institute of Medicine Committee on the Future of Emergency Care in the US Health System. Hospital-based Emergency Care: At the Breaking Point. Washington, DC: Institute of Medicine Committee on the Future of Emergency Care in the US Health System; 2006. https://www.nap.edu/Catalog/11621/hospital-based-emergencycare-at-the-breaking-point. Accessed February 12, 2019.

34. Yealy DM, Greene T\}, Hobbs GD. Underrecognition of cervical Neisseria gonorrhoeae and Chlamydia trachomatis infections in the emergency department. Academic Emergency Medicine. 1997;4(10):962-967.

35. Finelli L, Schillinger JA, Wasserheit JN. Are emergency departments the next frontier for sexually transmitted disease screening? Sexually Transmitted Diseases.

2001;28(1):40-42.

36. Gudka S, Afuwape FE, Wong B, Yow XL, Anderson C, Clifford RM. Chlamydia screening interventions from community pharmacies: a systematic review. Sexual Health. 2013;10(3):229-239. 
37. Australian Government Department of Health and Ageing. To Develop and Pilot a Best Practice Community Pharmacy Chlamydia Screening model. Canberra, Australia: Australian Government Department of Health and Ageing; 2010. http://6cpa.com.au/wp-content/uploads/To-Develop-and-Pilot-a-Best-PracticeCommunity-Pharmacy-Chlamydia-Screening-Model-final-report-includingappendices-.pdf. Accessed February 12, 2019.

38. Watson M, NHS Grampian Chlamydia Study Working Group. Final report: chlamydia testing in community and primary care settings. Aberdeen, United Kingdom: NHS Grampian; 2008.

39. Bloomfield PJ, Kent C, Campbell D, Hanbrook L, Klausner JD. Community-based chlamydia and gonorrhea screening through the United States mail, San Francisco. Sexually Transmitted Diseases. 2002;29(5):294-297.

40. Department of Health. Pharmacy chlamydia screening pathfinder evaluation (PCSPf). London, United Kingdom: Department of Health; 2007.

41. Taylor KL, Clifford RM, Marshall L. Acceptance of a chlamydia screening program in community pharmacies. Journal of Pharmacy Practice and Research. $2007 ; 37(4) ; 287-291$.

42. Baraitser P, Pearce V, Holmes J, Horne N, Boynton P. Chlamydia testing in community pharmacies: evaluation of a feasibility pilot in south east London. BMJ Quality \& Safety. 2007;16(4):303-307.

43. Bernstein KT, Chow JM, Pathela P, Gift TL. Bacterial sexually transmitted disease screening outside the clinic-implications for the modern sexually transmitted disease program. Sexually Transmitted Diseases. 2016;43(2 Suppl 1):S42. 
44. Grimley DM, Annang L, Lewis I, et al. Sexually transmitted infections among urban shelter clients. Sexually Transmitted Diseases. 2006;33(11):666-669.

45. Johnson CC, Jones EH, Goldberg M, Asbel LE, Salmon ME, Waller CL. Screening for Chlamydia trachomatis and Neisseria gonorrhoeae among adolescents in Family Court, Philadelphia, Pennsylvania. Sexually Transmitted Diseases. 2008;35(11):S24S27.

46. Ellen JM, Bonu S, Arruda JS, Ward MA, Vogel R. Comparison of clients of a mobile health van and a traditional STD clinic. Journal of Acquired Immune Deficiency Syndromes 2003;32(4):388-393.

47. Jamil MS, Bauer HM, Hocking JS, et al. Chlamydia screening strategies and outcomes in educational settings: A systematic reviêิ. Sexually Transmitted Diseases. 2014;41(3):180-187.

48. Gaydos CA, Wright C, Wood BJ, Waterfield G, Hobson S, Quinn TC. Chlamydia trachomatis reinfection rates anong female adolescents seeking rescreening in school-based health centers. Sexually Transmitted Diseases. 2008;35(3):233.

49. Nsuami MJ, Taylor SN, Smith BS, Martin DH. Increases in gonorrhea among high school students following hurricane Katrina. Sexually Transmitted Infections. 2009;85(3):194-198.

50. Bowden FJ, O'Keefe EJ, Primrose R, Currie MJ. Sexually transmitted infections, bloodborne viruses and risk behaviour in an Australian senior high school populationThe SHLiRP study. Sexual Health. 2005;2(4):229-236. 
51. Buhrer-Skinner M, Muller R, Menon A, Gordon R. Novel approach to an effective community-based chlamydia screening program within the routine operation of a primary healthcare service. Sexual Health. 2009;6(1):51-56.

52. Hengel B, Jamil MS, Mein JK, Maher L, Kaldor JM, Guy RJ. Outreach for chlamydia and gonorrhoea screening: a systematic review of strategies and outcomes [electronic article]. BMC Public Health. 2013;13(1):1040.

53. Gold J, Hocking J, Hellard M. The feasibility of recruiting young men in rural areas from community football clubs for STI screening. Australian and New Zealand journal of public health. 2007;31(3):243-246.

54. Götz H, Veldhuijzen I, Ossewaarde J, de Zwart O, Richardus JH. Chlamydia trachomatis infections in multi-ethnic urbanyouth: a pilot combining STI health education and outreach testing in Rotterdam, Netherlands. Sexually Transmitted Infections. 2006;82(2):148-152.

55. Ginige S, Fairley CK, Hocking S S, Bowden FJ, Chen MY. Interventions for increasing chlamydia screening in primary care: a review. BMC Public Health [electronic article]. 2007;7(1):95.

56. Armstrong B, Kinn S, Scoular A, Wilson P. Shared care in the management of genital Chlamydia trachomatis infection in primary care. Sexually Transmitted Infections. 2003;79(5):369-370.

57. Allison JJ, Kiefe CI, Wall T, et al. Multicomponent Internet continuing medical education to promote chlamydia screening. American Journal of Preventive Medicine. 2005;28(3):285-290. 
58. Centers for Disease Control and Prevention. Recommendations for the prevention and management of Chlamydia trachomatis infections. 1993. Atlanta, GA: Centers for Disease Control and Prevention;1993.

https://www.cdc.gov/mmwr/preview/mmwrhtml/00021622.htm. Accessed

February 12, 2019.

59. Centers for Disease Control and Prevention. Sexually Transmitted Diseases Treatment Guidelines, 2010. Atlanta, GA: Centers for Disease Control and Prevention; 2010.

https://www.cdc.gov/mmwr/preview/mmwrhtml/rr5912a1.htm. Accessed February 12, 2019.

60. Centers for Disease Control Prevention. Chlamydia screening practices of primarycare providers--Wake County, North Carolina, 1996. MMWR Morbidity and Mortality Weekly Report. 1997;46(35):819-822.

61. Madden M, Lenhart A, Duggan M, Cortesi S, Gasser U. Teens and Technology 2013. 2013; http://www.pewinternet.org/2013/03/13/teens-and-technology-2013/. Published March 13, 2013. Accessed February 12, 2019.

62. Dowshen N Lee S, Lehman BM, Castillo M, Mollen C. IknowUshould2: feasibility of a youth-driven social media campaign to promote STI and HIV testing among adólescents in Philadelphia. AIDS and Behavior. 2015;19(2):106-111.

63. Low N, Redmond S, Uusküla A, et al. Screening for genital chlamydia infection. Cochrane Database of Systematic Reviews [electronic article]. 2016;9(CD010866).

64. Nelson HD, Helfand M. Screening for chlamydial infection. American Journal of Preventive Medicine. 2001;20(3):95-107. 
65. Cao B, Gupta S, Wang J, et al. Social Media Interventions to Promote HIV Testing, Linkage, Adherence, and Retention: Systematic Review and Meta-Analysis [electronic article]. Journal of Medical Internet Research. 2017;19(11). e394

66. Unemo M, Bradshaw CS, Hocking JS, et al. Sexually transmitted infections: challenges ahead. The Lancet Infectious Diseases. 2017;17(8):e235-e279.

67. Ferreira A, Young T, Mathews C, Zunza M, Low N. Strategies for partner notification for sexually transmitted infections, including HIV [electronic article]. Cochrane Database of Systematic Reviews. 2013;10(CD002843)

68. Moher D, Liberati A, Tetzlaff J, Altman DG. Preferred reporting items for systematic reviews and meta-analyses: the PRISMA statement. Annals of internal medicine. 2009;151(4):264-9. 
Figure 1: The socio-ecological model

Figure 2: Preferred Reporting Items of Systematic Reviews and Meta-analyses (PRISMA) flow

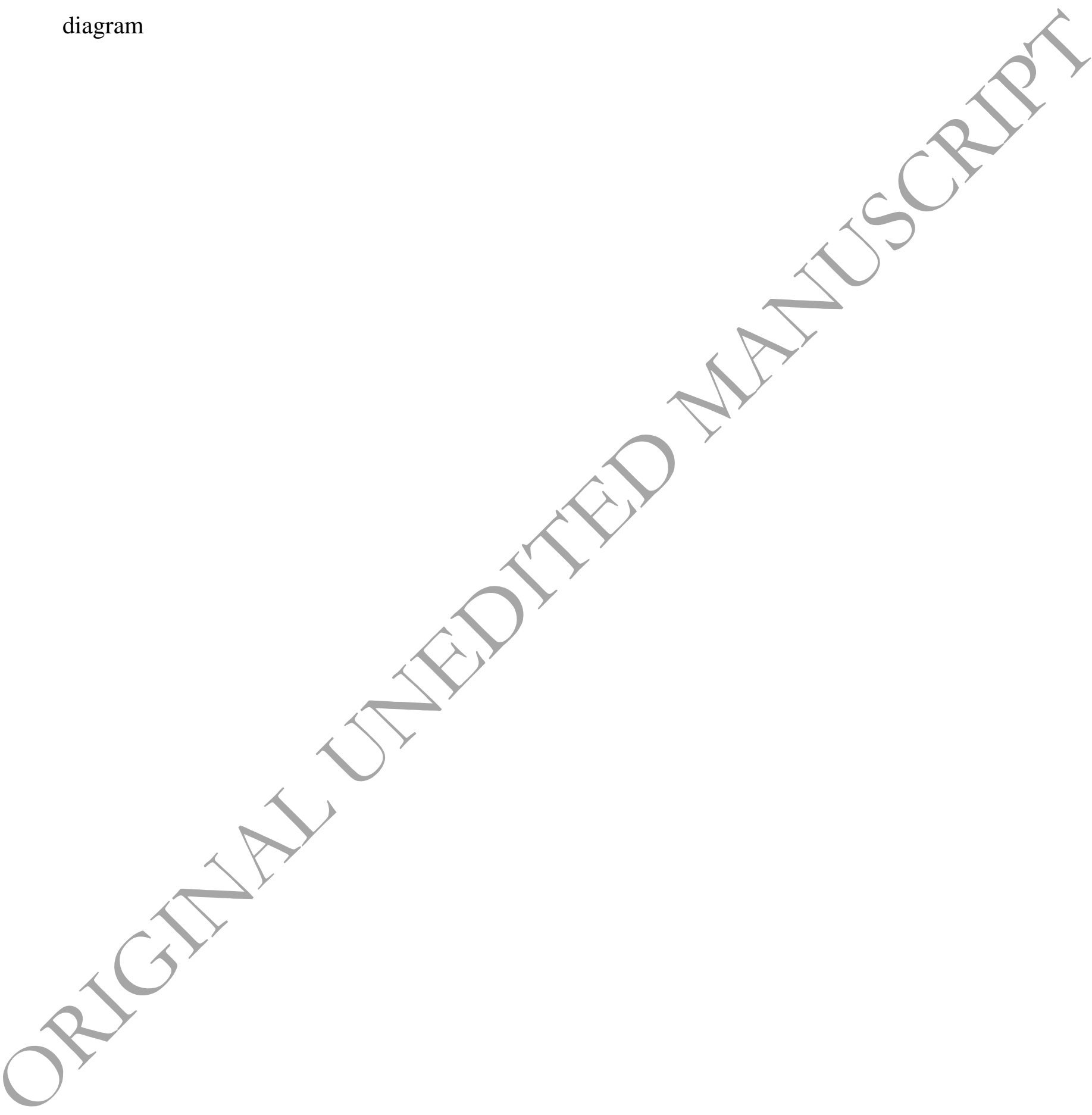


Figure 1.

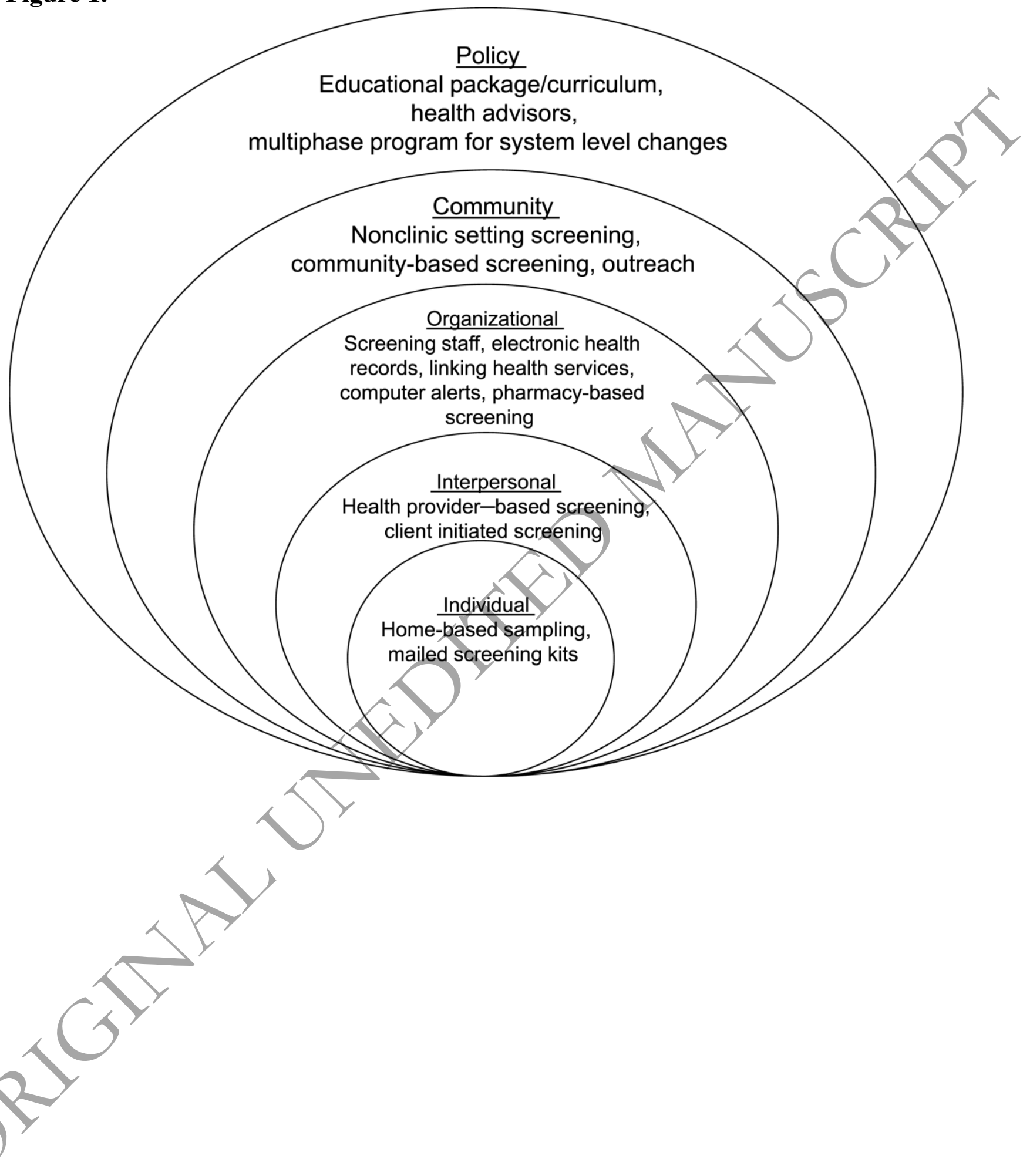


Figure 2.

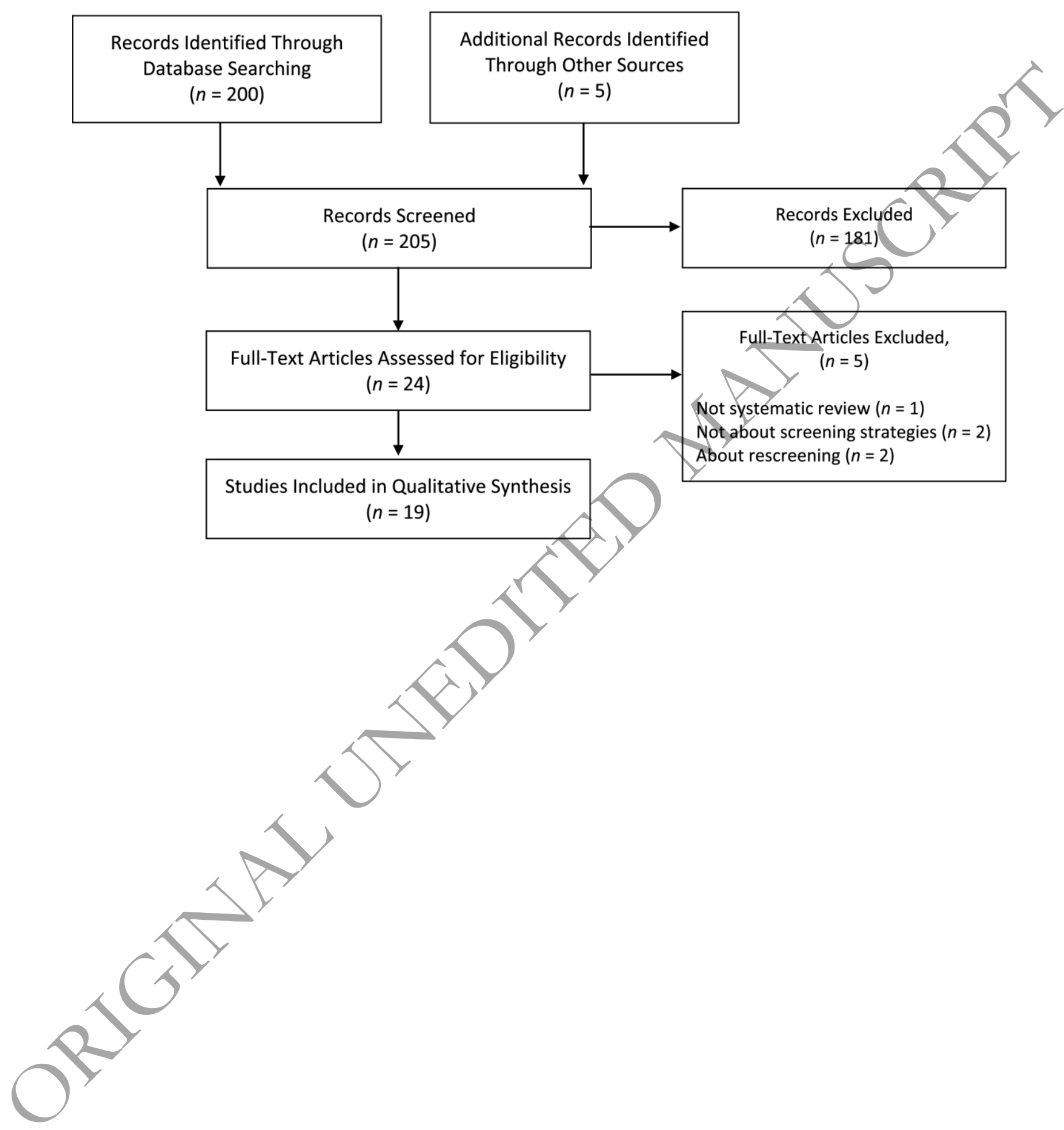

